\title{
QUALIFICAÇÃO DE PROFISSIONAIS DA SAÚDE PARA A ATENÇÃO ÀS MULHERES EM SITUAÇÃO DE VIOLÊNCIA SEXUAL
}

\author{
TRAINING OF HEALTH CARE PROFESSIONALS FOR THE CARE \\ OF WOMEN IN INSTANCES OF SEXUAL VIOLENCE
}

\author{
CUALIFICACIÓN DE PROFESIONALES DE LA SALUD PARA LA ATENCIÓN \\ DE MUJERES EN SITUACIÓN DE VIOLENCIA SEXUAL
}

Gracyelle Alves Remigio Moreira ${ }^{1}$

Kerma Márcia de Freitas ${ }^{2}$

Ludmila Fontenele Cavalcanti ${ }^{3}$

Luiza Jane Eyre de Souza Vieira ${ }^{4}$

Raimunda Magalhães da Silva ${ }^{5}$

Resumo Analisou-se a qualificação de profissionais da saúde para a atenção às mulheres em situação de violência sexual em duas capitais brasileiras, em estudo qualitativo envolvendo 140 profissionais de diferentes categorias de 18 instituições de saúde em Fortaleza e Rio de Janeiro. Entrevistas semiestruturadas foram realizadas e o material empírico foi analisado segundo a técnica de análise de conteúdo. Lacunas na formação dos profissionais decorrentes da insuficiente abordagem do tema durante a graduação dos cursos da área da saúde foram observadas nos dois municípios. A fragilidade da qualificação profissional para esse tipo de atuação foi reforçada por lacunas nas ações de capacitação. No município do Rio de Janeiro essas ações mostraram-se mais frequentes e contínuas. Importante a inclusão do tema na formação profissional, a ampliação de processos de capacitação e a atualização das equipes de saúde nos serviços. Tais medidas podem viabilizar um entendimento crítico sobre o fenômeno, contribuindo para implementar intervenções diferenciadas.

Palavras-chave violência contra a mulher; violência sexual; formação profissional em saúde; educação permanente.
Abstract We analyzed the training of health care professional for the care of women in instances of sexual violence in two Brazilian state capitals, in a qualitative study with 140 professionals of different categories working in 18 health care institutions in the cities of Fortaleza and Rio de Janeiro. Semi-structured interviews were performed, and the empirical material was analyzed according to the content analysis technique. Gaps in the training of the professionals due to an insufficient approach of this topic during the undergraduate studies in the field of health were observed in both municipalities. The weakness of the professional training for this kind of practice was reinforced by gaps in the training actions. In the city of Rio de Janeiro, these actions were more frequent and continuous. The inclusion of this topic in the professional training, the expansion of training processes, and the continuing education of the health care teams in the services are important. Such measures will make viable a critical understanding of this phenomenon, contributing to the implementation of diverse interventions.

Keywords violence against women; sexual violence; professional training in health; permanent education. 


\section{Introdução}

O United Nations Development Fund for Women (Unifem) declara que a violência contra a mulher é prioritária para a saúde pública, sendo também uma questão de paz e segurança; a organização se compromete a subsidiar os países para que os funcionários públicos adquiram conhecimentos e habilidades técnicas para enfrentar todo e qualquer tipo de violência contra as mulheres, considerando a multidimensionalidade que a circunscreve (Unifem, 2011).

A violência sexual é uma das formas mais hediondas de violência de gênero e violação de direitos humanos, sexuais e reprodutivos, conformando-se como um dos principais indicadores de discriminação contra a mulher (Contreras et al., 2010; Unifem, 2011). Estudos multipaíses registram que a violência sexual afeta milhões de mulheres anualmente. Informam que uma a cada três mulheres é abusada sexualmente antes de completar 18 anos, que o parceiro íntimo é o principal agressor e que o domicílio é o local mais comum dessas ocorrências (García-Moreno et al., 2005; OMS, 2002).

O fenômeno tem alcançado maior visibilidade, reafirmando-se como objeto de estudo nas investigações acadêmicas e mobilizando as nações signatárias da Convenção pela Eliminação de Todas as Formas de Discriminação Contra a Mulher (CEDAW, na sigla em inglês ) e da Convenção Interamericana para Prevenir, Punir e Erradicar a Violência Contra a Mulher (Convenção Belém do Pará) na concentração de esforços para o seu enfrentamento (Unifem, 2011; OMS, 2002; Black, 2011). Nessa trajetória de embates e conquistas, percebemse avanços no campo da violência sexual contra a mulher no Brasil, seja no âmbito legal ou no das políticas públicas, com a criação de dispositivos para seu enfrentamento, assim como a organização de práticas e serviços específicos.

De acordo com o Ministério da Saúde, os trabalhadores do setor saúde devem estar capacitados e aptos para adotar as medidas protetoras recomendadas nos protocolos técnicos e prestar um atendimento humanizado, livre de julgamentos morais e de crenças pessoais (Brasil, 2012). Nesse contexto, as três esferas de governo despendem recursos financeiros e técnicos em capacitação sobre o tema. Entre outras iniciativas em curso, as instâncias responsáveis pela formação desses profissionais têm assegurado a realização dessas capacitações com formatos em educação à distância, materiais instrutivos, normativos e educativos estão disponíveis (on line) e são de fácil acesso. Nessa compreensão, esses empreendimentos contribuem para ancorar a prática profissional na atenção à saúde dessas mulheres.

Em contraponto, os profissionais da saúde asseguram que não se sentem preparados para atender a essa demanda, visto que permanecem lacunas sobre concepções teóricas e estratégias operacionais, decorrentes de aborda- 
gens superficiais na formação acadêmica e fragilidades na implementação de uma educação permanente no contexto de práticas profissionais. Desse modo, persistem os desafios à efetivação dos dispositivos técnicos e também legais de garantia dos direitos de cidadania das mulheres.

Vasta literatura reitera que os profissionais da gestão e atenção à saúde não se consideram aptos para ofertar uma atenção qualificada e resolutiva às mulheres expostas à violência sexual que recorrem a esses serviços, ainda que se reconheça a sua exigência legal (Vieira, Padoin e Landerdahl, 2009; Kiss e Schraiber, 2011; Alcaraz et al., 2014).

Diante dessa conjuntura e da obrigatoriedade de que todos os serviços de saúde ofertem uma atenção qualificada a essas mulheres (Brasil, 2013), o cenário impõe amplos debates que instituam nos currículos conteúdos sobre as manifestações da violência contra a mulher e suas repercussões e que possibilitem análises sistemáticas sobre como as ações de capacitação estão sendo conduzidas e o efetivo monitoramento sobre a reorientação na oferta da atenção à saúde às mulheres expostas à violência sexual.

Desse modo, este estudo assumiu como objetivo analisar a qualificação de profissionais da saúde para a atenção às mulheres em situação de violência sexual em duas capitais brasileiras.

\section{Trajetória investigativa}

Estudo qualitativo, que integrou uma pesquisa ${ }^{6}$ mais abrangente que analisou a atenção em saúde às mulheres em situação de violência sexual em duas capitais brasileiras, Fortaleza e Rio de Janeiro, com a participação de 140 profissionais da atenção e 34 da gestão. Este artigo se reporta aos aspectos da qualificação dos profissionais da saúde para a oferta da atenção a essas mulheres, compreendendo a abordagem do tema no percurso da formação acadêmica e em atividades de capacitação no decorrer da prática laboral.

O cenário da pesquisa foram os serviços de atenção secundária e terciária das redes municipais de saúde de Fortaleza e do Rio de Janeiro, abrangendo nove serviços em cada capital. A escolha desses dois municípios embasou-se no fato de representarem contextos culturais distintos, pertencentes a polos diferenciados no tocante aos indicadores sociais e de violência (Cavalcanti et al., 2015). Segundo a Pesquisa de Informações Básicas Municipais (Munic), os serviços especializados que integram a rede de atenção às mulheres em situação de violência sexual concentram-se na região Sudeste e a região Nordeste conta com o menor número de equipamentos (IBGE, 2015). No quadro das violências de gênero perpetradas contra mulheres, a taxa de homicídio é 10,4 por 100.000 mulheres em Fortaleza (4a posição no país) e de 3,4 por 100.000 mulheres no Rio de Janeiro (26 a posição no país) (Waiselfisz, 2015). 
O estudo nessas capitais permitiu conhecer o modo como o tema violência sexual contra a mulher é abordado na qualificação dos trabalhadores da saúde em cenários distintos.

Participaram da pesquisa profissionais da saúde de nível superior, integrantes da equipe multiprofissional, inseridos na atenção às mulheres em situação de violência sexual nos serviços selecionados. Ao todo, entrevistaram-se 68 profissionais em Fortaleza (19 assistentes sociais, 19 enfermeiros, 17 médicos, 12 psicólogos e uma pedagoga) e 72 no Rio de Janeiro (21 médicos, 20 enfermeiros, 18 assistentes sociais e 13 psicólogos).

A constituição do conjunto de participantes se baseou na representatividade de diversas categorias profissionais em cada instituição investigada, na experiência no atendimento a essas mulheres e na indicação interpessoal entre os trabalhadores das instituições (snow ball) com maior experiência sobre a questão em foco (Vieira et al., 2016). Para definição do tamanho da amostra, considerou-se um número aceitável de interlocutores que permitisse a reincidência e complementaridade das informações. Entrevistar pessoas que integram um grupo (profissionais da saúde) sinaliza, ao mesmo tempo, uma declaração pessoal e coletiva (Minayo, 2017).

A coleta dos dados se deu entre agosto de 2013 e janeiro de 2014, por meio de entrevista semiestruturada com questões sobre caracterização e formação profissional, inserção institucional, qualificação acerca do tema e as ações que compõem a atenção em saúde de mulheres em situação de violência sexual.

As entrevistas foram realizadas individualmente e em local reservado, em dias e horários previamente acordados com as instituições e os profissionais. As gravações tiveram média de 30 minutos por entrevista, sendo posteriormente transcritas na íntegra e, como forma de preservar o anonimato dos participantes, codificadas com as siglas PF (Profissional Fortaleza), numeradas de 1 a 68, e PR (Profissional Rio de Janeiro), numeradas de 1 a 72.

Para a análise dos dados obtidos por meio das entrevistas, inicialmente procedeu-se à organização do material, que foi decomposto em partes correspondentes aos itens abordados no roteiro e às respectivas falas dos participantes. Utilizou-se a técnica de análise de conteúdo na modalidade temática (Minayo, 2013), guiada pela trajetória analítica proposta por Gomes (2013), para análise do conjunto de dados. Após leitura detalhada das falas e da atribuição dos sentidos indicados pelos participantes, o material foi reagrupado em núcleos centrais mais abrangentes: inserção do tema nas matrizes curriculares; capacitação para a atenção às mulheres em situação de violência sexual na prática profissional.

A pesquisa, resultado de tese de doutorado, foi aprovada pelo Comitê de Ética em Pesquisa da Secretaria Municipal de Saúde e Defesa Civil do Rio de Janeiro, mediante o parecer n. 45A/2013. 


\section{Predominância feminina na atenção às mulheres em situação de violência sexual}

Em Fortaleza, predominaram os profissionais do sexo feminino, na faixa etária de 45 a 69 anos, que se declararam unidos (em casamento formal ou união estável) e de religião católica. O fato de a maioria dos participantes ser do sexo feminino confirma a maior inserção das mulheres nesse tipo de atuação e o predomínio feminino na ocupação de cargos no cenário da saúde, principalmente na atenção à saúde da mulher (Alcaraz et al., 2014; Sousa et al., 2015), o que significa que, neste estudo, predomina uma concepção feminina sobre a qualificação profissional para atuação nessa área.

Em relação à formação e atuação profissional, a maior parte dos entrevistados formou-se em instituições públicas, havia concluído a graduação entre 11 e 25 anos atrás, era pós-graduada e atuava na instituição analisada havia pelo menos cinco anos.

No Rio de Janeiro, também sobressaíram os profissionais do sexo feminino, que se declararam unidos (casamento formal ou união estável) e de religião católica. Houve maior participação da faixa etária de 25 a 49 anos. A maioria dos participantes realizou o curso de graduação em instituições privadas, estava formada há entre cinco e 20 anos, era pós-graduada e atuava no serviço de saúde investigado há menos de cinco anos.

\section{Inserção do tema nas matrizes curriculares}

A abordagem do tema violência sexual contra a mulher e atuação do setor saúde frente ao fenômeno foi mencionada como ausente ou insuficiente durante a graduação por grande parte dos profissionais da saúde de Fortaleza e do Rio de Janeiro, o que evidencia distanciamento entre a questão e as matrizes curriculares. Alguns entrevistados enfatizaram que o assunto não foi trabalhado nos cursos da área da saúde. E revelaram que seu primeiro contato com a questão aconteceu, muitas vezes, na prática profissional em serviços de saúde ou quando, por iniciativa própria, buscaram diferentes formas de capacitação.

Nenhuma referência foi feita a esse assunto na minha graduação (...). Por eu ter trabalhado 16 anos em outra maternidade foi que eu tive contato com essa realidade e busquei de alguma forma me capacitar para fazer esse tipo de atendimento (PF64).

Não ouvi falar de violência sexual durante minha graduação (...). Sobre violência sexual fui preparada na prática mesmo, no dia a dia do trabalho (PR21). 
Entre os profissionais graduados há mais de vinte anos, a violência sexual contra a mulher, apesar de ser um problema que acompanha a história da humanidade, foi referida como um tema recente, não abordado na graduação em virtude de que, na época, o fenômeno não tinha visibilidade e não era priorizado pelas políticas públicas, como vem ocorrendo nos últimos anos:

Como já faz muito tempo que eu fiz a graduação, o tema violência sexual contra a mulher passava muito despercebido. Sempre existiu! Mas não tinha esse tipo de abordagem (PF54).

A violência sexual está sendo mais debatida de uns tempos para cá. Quando eu me formei não era (...). Agora que estão tendo alguma preocupação (PR57).

Do mesmo modo, também se percebeu nos relatos de participantes graduados há menos de cinco anos que a temática ainda se mantém pouca explorada na formação superior.

A escassez, na formação acadêmica, de preparação voltada para a questão se traduz em uma atuação profissional despreparada e incompleta diante dos casos de violência sexual. Os próprios participantes reconheceram que, devido a essa lacuna, ainda não conseguiam trabalhar em uma perspectiva integral, emancipatória e intersetorial. A precária qualificação torna-se um elemento complicador no direcionamento e na implementação de políticas públicas, e pode repercutir na falta de reconhecimento da problemática como campo de atuação do setor saúde.

Pesquisas, internacionais e nacionais, destacam o baixo percentual de estudantes e profissionais da saúde, sobretudo nos cursos de medicina, enfermagem e odontologia, que tiveram aulas sobre o assunto durante a graduação. Esses estudos também demonstram a ausência de abordagem acerca dos protocolos de atendimento e as fragilidades no tocante à habilidade para manejar os casos (Vicente e Vieira, 2009; Rosa et al., 2010; Alcaraz et al., 2014).

De acordo com Vieira, Padoin e Landerdahl (2009), os profissionais, em geral, saem das universidades sem que estejam preparados para enfrentar as demandas implícitas, as quais não são declaradas pelos usuários, o que recorrentemente acontece nas situações de violência que se apresentam aos serviços de saúde. Casos velados, sem sinais físicos visíveis, manifestam-se como empecilhos para o reconhecimento da violência. Guedes, Nogueira e Camargo Jr. (2009) argumentam que a falta de habilidade para desvelar o não aparente está relacionada com a formação baseada no modelo biomédico hegemônico, que não valoriza os aspectos subjetivos e a singularidade do sofrimento humano. 
Os entrevistados deste estudo que reconheceram a abordagem do tema em sua graduação a consideraram incipiente, passageira e inespecífica. O enfoque na questão foi julgado insatisfatório, esvaziado, sem conexão da teoria com as atividades práticas, não proporcionando a apreensão do conhecimento em virtude da superficialidade com que o assunto foi discutido. Os médicos dos dois municípios mencionaram que em sua graduação a abordagem da violência sexual contra a mulher se pautou no modelo clínico, tendo como base o modelo de atuação saúde-doença-conduta, direcionada para o diagnóstico da violência, seus sinais e sintomas e o tratamento das infecções sexualmente transmissíveis (ISTs).

Durante a graduação de medicina eles têm uma preocupação mais técnica. Existe uma preocupação maior com a questão das doenças que a gente tem que fazer a profilaxia, quais as lesões ginecológicas ou anais (...). Toda discussão é a respeito das questões técnicas, físicas, patológicas. Não passa pelas questões mais amplas da violência sexual, que eu acho até mais importante (PR46).

Percebe-se que o enquadramento dado a essa discussão não abrange aspectos fundamentais para a compreensão e abordagem da violência contra a mulher, como as questões de gênero. A preponderância do perfil clínicobiológico, substancialmente incrustado na estrutura curricular dos cursos da área da saúde, vai de encontro às características preconizadas pelo modo de produzir saúde no Sistema Único de Saúde (SUS) e não responde adequadamente à complexidade das situações de violência (Saldanha et al., 2014).

A reprodução de práticas tradicionais, fragmentadas e isoladas, fundadas na lógica tecnicista, voltadas para as partes do todo, restringe a atuação diante da problemática e não contempla as necessidades das usuárias. A literatura reitera essas evidências, atestando que a inclusão e o tratamento da temática na formação dos profissionais da saúde não são efetivos, visto que a compreensão da violência demanda processos de reflexão e aquisição de conhecimentos diferenciados, que incluem a sua dimensão político-social (Hamberger, 2007; Berger, 2011; Pedrosa e Spink, 2011).

Em um estudo acerca do ensino, na formação médica, sobre atenção a pessoas em situação de violência por parceiros íntimos, Hamberger (2007) sublinha que o frequente isolamento entre as atividades que envolvem o tema e as demais atividades e conteúdos temáticos que integram a matriz curricular é um limitador na formação, o que tem limitado, também, a habilitação para tal atuação.

Berger (2011) acrescenta que pouco se tem preparado os profissionais da saúde para a abordagem de temas sensíveis e que envolvem forte carga mo- 
ral, que demandam lidar com questões sociais complexas, como a violência sexual e o aborto legal, com suas próprias percepções e sentimentos. Bosi e colaboradores (2000, p. 43) denominam essa invisibilidade das questões que extrapolam as fronteiras da prática pautada no modelo biomédico de "silêncio curricular". O silêncio, no entanto, é mais do que curricular, pois há também o silenciamento dos debates sociais.

Nos âmbitos político-institucionais e paradigmáticos da produção do conhecimento, esse silêncio é reforçado pela concepção bancária da educação, assim como por um processo de trabalho em saúde centrado na figura do médico, que inviabiliza a implementação de rotinas respaldadas na ação coletiva que definam e articulem funções dentro de uma equipe multidisciplinar e da rede de serviços (Berger, 2011).

Nesse caminho, assim como observado na presente pesquisa, a violência sexual é, quase sempre, abordada de modo fragmentado e pontual, dissociado das ações nos serviços de saúde e de uma discussão que se converta em apropriações concretas de conhecimento, que reverbere em intervenções diferenciadas frente às mulheres atendidas.

Embora com menor expressão, alguns entrevistados referiram que a temática foi abordada de forma transversal em disciplinas, sobretudo nos cursos de psicologia e serviço social. O estágio curricular e a participação em pesquisas, principalmente para os profissionais do Rio de Janeiro, se configuraram como espaços favoráveis à vivência e internalização da atenção às mulheres em situação de violência sexual, propiciando a aquisição de conhecimentos e a adoção de posturas diante da questão.

Eu fiz um estágio em ginecologia em um hospital federal no Rio de Janeiro e a gente trabalhava diretamente com isso também. Tinha um ambulatório em que a gente acompanhava os casos de violência sexual (PR48).

Os cursos de psicologia e de serviço social, por tratarem com mais ênfase os referenciais das ciências humanas e sociais em suas matrizes curriculares, abordam o tema considerando em maior medida os aspectos históricos, sociais, psicológicos, culturais e dos direitos humanos que o permeiam. Essa abordagem enseja uma visão ampliada do fenômeno, que viabiliza um entendimento crítico sobre a violência sexual contra a mulher.

A proposta do SUS para a superação do modelo clássico de atenção à saúde da mulher incorpora o conceito ampliado de saúde, comportando a perspectiva dos determinantes sociais da saúde, na qual o ser humano é compreendido como sujeito de direito e o processo saúde-doença é visto como produto da relação entre a dimensão social e a biológica. Pensar essa inter-relação é re- 
conhecer a existência das iniquidades e os meios para superá-las, visando à promoção da saúde (Freitas, Oliveira e Silva, 2013).

As universidades devem direcionar suas ações para as necessidades da sociedade, aprofundando questionamentos sobre a dinâmica dos problemas sociais, assim como enfatizar formas de atuação que considerem a integralidade na atenção ao ser humano.

As mudanças na formação dos trabalhadores da saúde são indispensáveis para a atenção às pessoas em situação de violência nos serviços, por demandarem transformação das concepções e práticas dos futuros profissionais, ainda bastante centradas no enfoque biomédico. O distanciamento entre a academia e as questões sociais induz uma atuação deslocada dos fundamentos que norteiam as políticas públicas (Pedrosa e Spink, 2011).

Realça-se, nesse caminho, a responsabilidade das instituições formadoras em preparar os futuros profissionais para atuar diante da questão compreendendo a violência contra a mulher no âmbito da saúde e reconhecendo seu papel social na identificação e assistência articulada com outros setores (Vieira, Padoin e Landerdahl, 2009). Esforços direcionados ao entendimento da dinâmica desse problema social complexo permitem um processo de reconstrução que favorece a sensibilização para o tema e o aprendizado de formas de intervenção mais apropriadas aos casos (Kiss e Schraiber, 2011).

\section{Capacitação para a atenção às mulheres em situação de violência sexual na prática profissional}

Alguns autores advogam que em razão da crescente diversidade de informações e da necessidade de conhecimento nas diversas áreas, para a educação formal torna-se uma tarefa árdua garantir uma formação plena do sujeito. Como alternativa, indicam a educação permanente como instância de aprendizagem contínua que promove o desenvolvimento do sujeito na busca da competência pessoal, profissional e social (Paschoal, Mantovani e Méier, 2007). Diante das lacunas herdadas da formação e da necessidade de atualização, ações de capacitação sobre a atenção às mulheres em situação de violência sexual se mostram indispensáveis.

A aquisição de informações e conhecimento sobre a questão, para muitos participantes deste estudo, só veio a ocorrer na prática profissional. Cursos, treinamentos, atividades de capacitação, avaliações de competências e seminários acerca do tema foram realizados por alguns profissionais. Esses mecanismos de formação, quase sempre, nos dois cenários pesquisados, configuraram-se em eventos pontuais proporcionados pelos governos municipal, estadual e federal (Ministério da Saúde) e trataram de aspectos como a anticoncepção de 
emergência, as profilaxias das ISTs, o aborto legal, o fluxo de atendimento, a notificação dos casos e os encaminhamentos necessários.

A Secretaria Municipal de Saúde fazia algumas reuniões e cursos rápidos com a gente. Nesses cursos rápidos, reuniões e avaliações de competência, a gente foi aumentando o conhecimento, o que ofereceu mais condições para a gente avaliar melhor e ter uma postura melhor diante disso (PF6).

Quando entrei, em 1999, a gente já teve uma capacitação, e a partir daí o serviço começou a ampliar esses treinamentos para outras maternidades e para as grandes emergências do estado. Como esta instituição foi a primeira a atender esses casos, os treinamentos foram feitos pelo pessoal daqui. Eu recebi treinamento da instituição e da Secretaria de Saúde (PR2).

A participação em atividades de capacitação apresentou aspectos distintos nas duas capitais. No Rio de Janeiro os investimentos na capacitação das equipes de saúde foram mais regulares e abrangentes desde o ano 2000, ao passo que em Fortaleza as ações de capacitação foram dirigidas à implementação de serviços, com discussões voltadas para a sensibilização de gestores e profissionais, e definição dos serviços de referência para a rede de atenção. O que pode estar associado à recente trajetória de implementação da atenção às mulheres em situação de violência sexual no conjunto de unidades da rede pública de saúde em Fortaleza (Cavalcanti et al., 2015).

Apesar dos investimentos identificados por esferas governamentais, percebeu-se nos dois municípios a incipiente oferta de capacitação por iniciativa das próprias instituições de saúde. Algumas ações foram mencionadas pelos entrevistados de hospitais com estrutura de maternidade, considerados de referência para este tipo de atenção. Ainda assim, na maioria das situações tratou-se de iniciativas isoladas, não vinculadas a uma cultura institucional em que se valorize a educação permanente como política. Também se nota a valorização de conteúdos fundados na hegemonia biomédica e a parca abordagem dos aspectos históricos e socioculturais que sustentam o fenômeno. Observou-se ainda que os profissionais buscam, por iniciativa própria, meios de capacitação além das atividades programadas e ofertadas pelas instituições onde trabalham.

Em Fortaleza, foi marcante o fato de que nos hospitais voltados para o atendimento de urgência e emergência na área de traumato-ortopedia não se verificou qualquer empreendimento de ações de qualificação profissional sobre o fenômeno. Em alguns casos havia o incentivo da gestão municipal para atuação nesse âmbito, mas entraves institucionais não permitiram implementála. Em outros contextos, os participantes foram unânimes em afirmar que a 
questão não era abordada, o que atribuíam ao fato de esse tipo de atenção não constituir uma função do serviço:

Não estamos preparados para atender, nos colocamos como hospital de referência no atendimento à mulher vítima de violência, mas sem referência, sem condições de atendimento. Não adianta eu ter num plantão um que está preparado, que sabe orientar direitinho, no outro não ter condição de atender. O ideal era que houvesse uma formação boa, que a gente pudesse envolver toda a equipe (PF8).

Essas evidências sinalizam que as lacunas do conhecimento transcendem os limites das instituições formadoras e se estendem para a rotina das unidades de saúde, em virtude de a temática permanecer, muitas vezes, oculta na pauta de educação permanente dos serviços. De acordo com os relatos dos entrevistados, mesmo quando abordada a questão não exerce impacto suficiente para potencializar a qualificação da atenção e para dar aos profissionais mais segurança no desempenho das práticas direcionadas às mulheres em situação de violência sexual. Também se percebe que a equipe não era capacitada com um todo e que não ocorria a disseminação do conhecimento.

Esse hiato na formação se reflete substancialmente no exercício das funções. Sem capacitação para o enfrentamento da questão, alguns profissionais se solidarizam com a situação, desenvolvendo estratégias próprias e informais de condução do atendimento, mas uma parcela mais expressiva tende a se distanciar dos casos, negligenciando a atenção ou deixando que a violência passe despercebida.

A visibilidade da violência sexual contra as mulheres, o aumento da demanda dos casos nos serviços de saúde e o forte impacto do fenômeno sobre gestores e profissionais que se encarregam da organização dessa atenção exigem investimentos continuados e diversificados em educação permanente. $\mathrm{O}$ modelo hegemônico tradicional é insuficiente para dar conta da complexidade desse desafio, requerendo esforços para compartilhamento e exploração de estratégias e tecnologias que contribuam para o desenvolvimento da atuação profissional (Flores, Oliveira e Zocche, 2016).

As políticas públicas e os marcos legais que versam sobre o problema postulam que a capacitação constante dos recursos humanos que operacionalizam as ações é uma estratégia elementar para a implementação do que está preconizado (Brasil, 2011a, 2011b, 2012). Para viabilizá-la, as secretarias e instituições de Saúde devem assumir este papel, e planos de capacitação profissional devem ser incorporados nos programas de gestão.

A gestão institucional de cada unidade hospitalar, em parceria com as secretarias de Saúde, é responsável por prover sua força de trabalho de conhecimentos científicos e instrumentais de modo permanente, haja vista as 
modalidades de contratualização, renovação, absenteísmo e a precarização dos vínculos profissionais que têm caracterizado o setor saúde nos municípios investigados.

A educação permanente dos profissionais que participam das equipes é defendida como uma ferramenta elementar para a melhoria do acolhimento e da atenção às mulheres em situação de violência sexual nos serviços (García-Moreno et al., 2015; Stewart et al., 2015). A falta de informação sobre a legislação, as políticas e os marcos legais impõe obstáculos nesse processo, prejudicando a qualidade da atenção. Principalmente nos serviços hospitalares, onde a maioria dos profissionais trabalha em regime de plantão e inexiste uma equipe específica para atender a essa demanda, é primordial que as ações de capacitação alcancem todas as equipes. Isso potencializará o alinhamento das orientações e condutas, evitando a fragmentação e descontinuidade das práticas assistenciais.

A formação de prestadores de cuidados de saúde é central para qualquer estratégia de enfrentamento da violência. Bedone e Faúndes (2007) enfatizam que quanto mais profissionais estiverem capacitados para esse tipo de abordagem, melhores serão as condições de se oferecer uma atenção digna e desburocratizada. Esses autores ainda postulam que todos os profissionais devem ser igualmente capacitados e motivados a se sentirem peças importantes dentro da equipe.

Nessa perspectiva, os processos de educação permanente possibilitam trocas de conhecimento e o contínuo aprimoramento da equipe, se configurando em um espaço para reflexão e crescimento coletivo, fortalecendo as relações interpessoais e proporcionando maior integração da equipe; têm, portanto, potencial para transformar práticas e modificar o cenário de ações improvisadas e assistemáticas (Andrade et al., 2016).

Importante frisar que para se afastar uma atenção puramente técnica e descontextualizada, os processos de educação permanente não devem se limitar aos aspectos técnicos e a uma aproximação conceitual da violência. Almeida et al. (2011) propõem que os recursos humanos da saúde tenham formação de caráter político para além da dimensão curativa do processo de trabalho, trazendo para si parte da responsabilização pela transformação social relacionada à opressão de gênero. Sob essa ótica, as concepções de gênero devem ser consideradas na formação/capacitação dos profissionais sobre a violência contra a mulher, a fim de se construir novas formas de fazer o cuidado em saúde.

Ações de educação permanente precisam ser avaliadas sistematicamente, averiguando-se o alcance da formação, a metodologia empregada, a forma de explanação e o envolvimento dos que estão sendo capacitados, bem como seu impacto na transformação de realidades. Uma gestão atenta à dinâmica das mudanças que demandam reorganização de processos gerenciais pode 
identificar quando a prática clínica estiver sendo influenciada pela política institucional vigente sem sintonia com a realidade sociossanitária.

Importante mencionar algumas limitações do estudo que, no entanto, não comprometem a contribuição dos dados aqui elencados. A primeira está relacionada às entrevistas: supõe-se que os participantes, em alguns momentos, reproduzam nos depoimentos as suas próprias condutas ou as da instituição em que estão inseridos; neste caso, podem omitir fragilidades estruturais ou processuais para preservar a instituição ou se preservar, sobretudo com a disseminação de contratos terceirizados. A segunda é o fato de a coleta de dados nas instituições ter ocorrido em períodos diferentes. E, por fim, quanto às fontes, não houve triangulação dos dados e não se incluiu a análise da documentação institucional acerca das iniciativas de capacitação.

\section{Considerações finais}

A análise da qualificação de profissionais da saúde para a atenção às mulheres em situação de violência sexual revelou que a abordagem do tema pelas instituições de ensino acontece de forma incipiente até mesmo para os profissionais formados mais recentemente. As lacunas na formação dos profissionais decorrente da tímida abordagem da questão durante a graduação dos cursos da área da saúde foram observadas pelos profissionais da saúde em Fortaleza e no Rio de Janeiro.

A fragilidade da qualificação profissional para esse tipo de atuação se aprofundou com a falta de capacitação ou pelo fato de as iniciativas voltadas para este fim serem esporádicas, o que impossibilita que cumpram sua função principal, a transformação de práticas e realidades.

Apesar desses pontos de convergência, os resultados indicam diferenças entre os dois municípios em relação à oferta de ações de capacitação por parte das esferas governamentais. No Rio de Janeiro essas ações se mostram mais frequentes e contínuas do que em Fortaleza em virtude do fato de o processo de implementação das ações de atenção em saúde às mulheres em situação de violência sexual estar mais avançado.

As lacunas relativas à qualificação dos profissionais da saúde apontam para a necessidade de inclusão e aprofundamento desse tema na formação profissional. Torna-se primordial a integração, de fato, dos referenciais das ciências humanas e sociais nas matrizes curriculares dos cursos da saúde, de modo a viabilizar um entendimento crítico sobre o fenômeno que contribua para intervenções qualificadas.

Acresce-se a necessidade de investimento e ampliação de processos de capacitação e atualização das equipes de saúde nos serviços. Tais abordagens exigem um trabalho contínuo, sistemático e participativo, em sintonia com a 
dinâmica dos atendimentos, que propicie aos profissionais reflexões e discussões sistemáticas na busca de resoluções de impasses que alcancem mudanças paradigmáticas.

\section{Colaboradores}

Gracyelle Alves Remigio Moreira trabalhou na coleta, análise e interpretação dos dados e na redação do artigo. Kerma Márcia de Freitas colaborou na coleta e análise dos dados e na redação do texto. Ludmila Fontenele Cavalcanti foi responsável pela concepção e delineamento do estudo, análise dos dados, revisão crítica e aprovação da versão a ser publicada. Luiza Jane Eyre de Souza Vieira contribuiu na análise e interpretação dos dados, na redação final do artigo e na aprovação da versão a ser publicada. Raimunda Magalhães da Silva cooperou na análise dos dados, na revisão crítica e na aprovação da versão a ser publicada. Não há conflito de interesses.

Resumen Se analizó la cualificación de los profesionales de la salud para la atención de las mujeres en situación de violencia sexual en dos capitales brasileñas, en un estudio cualitativo abarcando 140 profesionales de diferentes categorías de 18 instituciones de salud en las ciudades de Fortaleza y Rio de Janeiro. Se realizaron entrevistas semiestructuradas y el material empírico se analizó de acuerdo con la técnica de análisis de contenido. En ambos municipios se observaron lagunas en la formación de los profesionales derivadas de un abordaje insuficiente del tema durante los estudios de grado en ciencias de la salud. La fragilidad de la cualificación profesional para este tipo de atención se vio reforzada por lagunas en las acciones de capacitación. En el municipio de Rio de Janeiro estas acciones demostraron una mayor frecuencia y continuidad. Es de suma importancia incluir este tema en la formación profesional, la ampliación de los procesos de capacitación y la actualización de los equipos de salud en los servicios de atención. Estas medidas pueden permitir una comprensión más crítica sobre el fenómeno, contribuyendo para implementar formas de intervención diferenciadas.

Palabras clave violencia contra la mujer; violencia sexual; formación profesional en salud; educación permanente. 


\section{Notas}

${ }^{1}$ Empresa Brasileira de Serviços Hospitalares, Maternidade Escola Assis Chateaubriand, Fortaleza, Ceará, Brasil.

< gracyremigio@gmail.com>

Correspondência: Empresa Brasileira de Serviços Hospitalares, Maternidade Escola Assis Chateaubriand (MEAC), Rua Coronel Nunes de Melo, s/n, Rodolfo Teófilo, CEP 60430270, Fortaleza, Ceará, Brasil.

${ }^{2}$ Faculdade Vale do Salgado, Icó, Ceará, Brasil.

<kerma@fvs.edu.br>

${ }^{3}$ Universidade Federal do Rio de Janeiro, Programa de Pós-Graduação em Serviço Social, Rio de Janeiro, Rio de janeiro, Brasil.

$<$ ludmila.ufrj@gmail.com>

${ }^{4}$ Universidade de Fortaleza, Programa de Pós-Graduação em Saúde Coletiva, Fortaleza, Ceará, Brasil.

$<$ janeeyre@unifor.br>

${ }^{5}$ Universidade de Fortaleza, Programa de Pós-Graduação em Saúde Coletiva, Fortaleza, Ceará, Brasil.

<rmsilva@unifor.br>

${ }^{6}$ A pesquisa foi financiada pelo Conselho Nacional de Desenvolvimento Científico e Tecnológico (CNPq) através do Edital MCTI/CNPq/SPM-PR/MDA n. 32/2012 - Relações de Gênero, Mulheres e Feminismos.

\section{Referências}

ALCARAZ, Carmen L. et al. Formación y detección de la violencia de género en la profesión sanitária. Revista de Enfermagem da UFSM, Santa Maria, v. 4, n. 1, p. 217-226, 2014.

ALMEIDA, Luana R. et al. A violência de gênero na concepção dos profissionais de saúde da atenção básica. Saúde em Debate, Rio de Janeiro, v. 35, n. 90, p. 396-404, 2011.

ANDRADE, Rebecca S. et al. Processo de trabalho em unidade de saúde da família e a educação permanente. Trabalho, Educação e Saúde, Rio de Janeiro, v. 14, n. 2, p. 505$521,2016$.
BEDONE, Aloísio J.; FAÚNDES, Aníbal. Atendimento integral às mulheres vítimas de violência sexual: Centro de Assistência Integral à Saúde da Mulher, Universidade Estadual de Campinas. Cadernos de Saúde Pública, Rio de Janeiro, v. 23, n. 2, p. 465-469, 2007.

BERGER, Sônia M. D. Violência entre parceiros íntimos: desafios no ensino e atenção em saúde. Revista Brasileira de Educação Médica, Rio de Janeiro, v. 35, n. 4, p. 526-534, 2011.

BLACK, Michele C. et al. Encuesta Nacional sobre Violencia Sexual y de Pareja. El informe sumario del 2010. Atlanta, GA: Centro Nacional 
para la Prevención y el Control de Lesiones, Centros para el Control y la Prevención de Enfermedades, 2011.8 p.

BOSI, Maria L. M. et al. Aborto provocado: o ponto de vista de acadêmicos de medicina de uma universidade pública. Cadernos de Saúde Coletiva, Rio de Janeiro, v. 8, n. 2, p. 41-54, 2000.

BRASIL. Presidência da República. Secretaria Nacional de Enfrentamento à Violência contra as Mulheres. Secretaria de Políticas para as Mulheres. Politica nacional de enfrentamento à violência contra as mulheres. Brasília: Secretaria de Políticas para as Mulheres, 2011a.

BRASIL. Secretaria de Políticas para as Mulheres. Pacto nacional de enfrentamento à violência contra as mulheres. Brasília: Secretaria de Políticas para as Mulheres. $2011 \mathrm{~b}$.

BRASIL. Ministério da Saúde. Secretaria de Atenção à Saúde. Departamento de Ações Programáticas Estratégicas. Prevenção e tratamento dos agravos resultantes da violência sexual contra mulheres e adolescentes: norma técnica. 3. ed. Brasília: Ministério da Saúde, 2012.

BRASIL. Lei 12.845, de 1 de agosto de 2013. Dispõe sobre o atendimento obrigatório e integral de pessoas em situação de violência sexual. Diário Oficial da República Federativa do Brasil, Brasília, DF, 2 ago. 2013. Seção 1, p. 1.

CAVALCANTI, Ludmila F. et al. Implementação da atenção em saúde às violências sexuais contra as mulheres em duas capitais brasileiras. Saúde em Debate, Rio de Janeiro, v. 39, n. 107, p. 1.079-1.091, 2015.

CONTRERAS, Juan M. et al. Violência sexual na América Latina e no Caribe: uma análise de dados secundários. Pretória: Iniciativa de Pesquisa sobre Violência Sexual, 2010.

FLORES, Giovana E.; OLIVEIRA, Dora L. L.; ZOCCHE, Denise A. A. Educação permanente no contexto hospitalar: a experiência que res- significa o cuidado em enfermagem. Trabalho, Educação e Saúde, Rio de Janeiro, v. 14, n. 2, p. 487-504, 2016.

FREITAS, Waglania M. F.; OLIVEIRA, Maria H. B.; SILVA, Ana T. M. C. Concepções dos profissionais da atenção básica à saúde acerca da abordagem da violência doméstica contra a mulher no processo de trabalho: necessidades (in)visíveis. Saúde em Debate, Rio de Janeiro, v. 37, n. 98, p. 457-466, 2013.

GARCÍA-MORENO, Claudia et al. Estudio Multipaís de la OMS sobre Salud de la Mujer y Violencia Doméstica contra la Mujer: primeros resultados sobre prevalencia, eventos relativos a la salud y respuestas de las mujeres a dicha violência. Resumen del informe. Ginebra: Organización Mundial de la Salud, 2005.

GARCÍA-MORENO, Claudia et al. The healthsystems response to violence against women. The Lancet, Genebra, v. 385, n. 9.977, p. 1.5671.579, 2015.

GOMES, Romeu. Análise e interpretação de dados de pesquisa qualitativa. In: MINAYO, Maria C. S.; DESLANDES, Suely F.; GOMES, Romeu. Pesquisa social: teoria, método e criatividade. 33. ed. Petrópolis: Vozes, 2013.

GUEDES, Carla R.; NOGUEIRA, Maria I.; CAMARGO JR, Kenneth R. Os sofredores de sintomas indefinidos: um desafio para a atenção médica. Physis: Revista de Saúde Coletiva, Rio de Janeiro, v. 19, n. 3, p. 797-815, 2009.

HAMBERGER, Kevin L. Preparing the next generation of physicians: medical school and residency-based Intimate partner violence curriculum and evaluation. Trauma, Violence \& Abuse, United States of America, v. 8, n. 2, p. 214-225, 2007.

IBGE (INSTITUTO BRASILEIRO DE GEOGRAFIA E ESTATÍSTICA). Perfil dos municípios brasileiros 2015. Rio de Janeiro: IBGE, 2015.

KISS, Ligia B.; SCHRAIBER, Lilia B. Temas médico-sociais e a intervenção em saúde: a 
violência contra mulheres no discurso dos profissionais. Ciência \& Saúde Coletiva, Rio de Janeiro, v. 16, n. 3, p. 1943-1952, 2011.

MINAYO, Maria C. S. O desafio do conhecimento: pesquisa qualitativa em saúde. 13. ed. São Paulo: Hucitec, 2013.

MINAYO, Maria C. S. Amostragem e saturação em pesquisa qualitativa: consensos e controvérsias. Pesquisa Qualitativa, São Paulo, v. 5, n. 7, p. 1-12, 2017.

OMS (ORGANIZAÇÃO MUNDIAL DA SAÚDE). Relatório mundial sobre violência e saúde. Genebra: OMS, 2002.

PASCHOAL, Amarílis S.; MANTOVANI, Maria F.; MEIER, Marineli J. Percepção da educação permanente, continuada e em serviço para enfermeiros de um hospital de ensino. Revista da Escola de Enfermagem da USP, São Paulo, v. 41, n. 3, p. 478-484, 2007.

PEDROSA, Claudia M.; SPINK, Mary J. P. A violência contra a mulher no cotidiano dos serviços de saúde: desafios para a formação médica. Saúde e Sociedade, São Paulo, v. 20, n. 1, p. 124-135, 2011.

ROSA, Roselia et al. Violência: conceito e vivência entre acadêmicos da área da saúde. Interface: Comunicação, Saúde, Educação, Botucatu, v. 14, n. 32, p. 81-90, 2010.

SALDANHA, Olinda M. de F. L. et al. Clínica-escola: apoio institucional inovador às práticas de gestão e atenção na saúde como parte da integração ensino-serviço. Interface: Comunicação, Saúde, Educação, Botucatu, v. 18, supl. 1, p. 1.053-1.062, 2014.

SOUSA, Maria H. et al. Preenchimento da notificação compulsória em serviços de saúde que atendem mulheres que sofrem violência sexual. Revista Brasileira de Epidemiologia, São Paulo, v. 18, n. 1, p. 94-107, 2015.

STEWART, Donna E. et al. Latin American and Caribbean countries' baseline clinical and policy guidelines for responding to intimate partner violence and sexual violence against women. BMC Public Health, London, v. 15, n. 665 , p. 1-6, 2015.

UNIFEM (FUNDO DE DESENVOLVIMENTO DAS NAÇÕES UNIDAS PARA A MULHER). $O M S$ : violência contra mulher é prioridade de saúde pública. 2011. Disponível em: <http:// www.unifem.org.br/003/00301015.asp?ttCD_ CHAVE $=129438>$ Acesso em: 4 dez. 2016.

VICENTE, Luciana M.; VIEIRA, Elisabeth M. $\mathrm{O}$ conhecimento sobre a violência de gênero entre estudantes de medicina e médicos residentes. Revista Brasileira de Educação Médica, Rio de Janeiro, v. 33, n. 1, p. 63-71, 2009.

VIEIRA, Letícia B.; PADOIN, Stela M. M.; LANDERDAHL, Maria C. A percepção de profissionais da saúde de um hospital sobre a violência contra as mulheres. Revista Gaúcha de Enfermagem, Porto Alegre, v. 30, n. 4, p. 609-616, 2009.

VIEIRA, Luiza J. E. S. et al. Protocolos na atenção à saúde de mulheres em situação de violência sexual sob a ótica de profissionais de saúde. Ciência \& Saúde Coletiva, Rio de Janeiro, v. 21, n. 12, p. 3.957-3.965, 2016.

WAISELFISZ, Julio J. (org.). Mapa da Violência 2015: homicídio de mulheres no Brasil. Brasília: Flacso Brasil, 2015.

Recebido em 02/03/2017. Aprovado em 05/03/2018. 